\title{
Multiple myeloma: the (r)evolution of current therapy and a glance into the future
}

\author{
Annamaria Gulla' and Kenneth C. Anderson \\ Division of Hematologic Neoplasia, Department of Medical Oncology, Jerome Lipper \\ Multiple Myeloma Center, Dana-Farber Cancer Institute, Harvard Medical School, Boston, \\ MA, USA
}

Haematologica 2020

Volume 105(10):2358-2367

\section{Correspondence:}

KENNETH C. ANDERSON

kenneth_anderson@dfci.harvard.edu

Received: June 10, 2020.

Accepted: July 16, 2020.

Pre-published: July 23, 2020.

doi:10.3324/haematol.2020.247015

(C)2020 Ferrata Storti Foundation

Material published in Haematologica is covered by copyright. All rights are reserved to the Ferrata Storti Foundation. Use of published material is allowed under the following terms and conditions:

https://creativecommons.org/licenses/by-nc/4.0/legalcode. Copies of published material are allowed for personal or internal use. Sharing published material for non-commercial purposes is subject to the following conditions:

https://creativecommons.org/licenses/by-nc/4.0/leǵalcode, sect. 3. Reproducing and sharing published material for commercial purposes is not allowed without permission in writing from the publisher.

\section{ABSTRACT}

$\mathrm{O}$ ver the past 20 years, the regulatory approval of several novel agents to treat multiple myeloma (MM) has prolonged median patient survival from 3 to 8-10 years. Increased understanding of $\mathrm{MM}$ biology has led to advances in diagnosis, prognosis, and response assessment, and has informed the development of targeted and immune agents. Here we provide an overview of the recent progress in MM, and highlight the most promising research areas to further improve patient outcome in the future.

\section{Introduction}

Remarkable progress in our understanding of the pathobiology of myeloma $(\mathrm{MM})$ has transformed the treatment paradigm and patient outcome. Preclinical studies have guided the discovery of more effective targeted therapies and informed clinical management. However, constitutive and ongoing genetic complexity and instability, coupled with the tumor promoting, immunosuppressive bone marrow (BM) microenvironment, remain an obstacle to cure. An estimated 32,270 new MM cases and 12,830 deaths in 2020 in the USA, ${ }^{1}$ coupled with a worldwide $126 \%$ increase in MM cases from 1990 to 2016, ${ }^{2}$ highlight the urgent need for novel therapies.

\section{Definition of disease and precursor stages}

Multiple myeloma is characterized by malignant plasma cells (PC) in the BM associated in most cases with monoclonal protein in serum and or urine; PC can also be detected in extramedullary sites and/or peripheral blood during progression of disease. ${ }^{3,4}$ Examination for MM-defining events allows for the discrimination between MM and its precursor stages, namely monoclonal gammopathy of undetermined significance (MGUS) and smoldering MM (SMM). ${ }^{5}$ Specifically, diagnosis of $\mathrm{MM}$ requires $10 \%$ or more $\mathrm{PC}$ in the $\mathrm{BM}$ plus one or more signs of end-organ damage including hypercalcemia, renal dysfunction, anemia, or bone disease (CRAB criteria). ${ }^{4}$ Even without $\mathrm{CRAB}$ features, patients who manifest MM-defining events including clonal BM PC $>60 \%$, serum : ratio $>100$ fold, and/or more than one bone focal lesion on magnetic resonance imaging (MRI) or positron emission tomography (PET)/computed tomography (CT) scan are also treated, as their risk of progression to symptomatic disease is approximately $80 \%$ at 2 years. ${ }^{5,6}$ Clinical manifestations of MM result from excessive production of monoclonal immunoglobulin protein by malignant PC in blood and/or urine, infiltration of BM by neoplastic clone, and aberrant cytokine secretion. ${ }^{4}$ MGUS patients are monitored for progression off all therapy, as their risk of progression overall is $1 \%$ yearly. The standard of practice is also to follow SMM patients expectantly off treatment, ${ }^{7,8}$ as the risk of progression is $10 \%$ per year in the first 5 years, dropping to $3 \%$ per year thereafter. Recently, the new "20-20-20" Mayo Clinic criteria have identified a high-risk (HR)-SMM subgroup (patients with two or more features including: BM PC infiltration $>20 \%$, monoclonal protein $>20 \mathrm{~g} / \mathrm{L}$ and FLC ratio $>20$ ) with a median time to progression of 29 months. ${ }^{9}$ The QuiRedex study showed that lenalidomide+dexamethasone treatment prolonged time to progression and overall survival (OS) in HR-SMM. ${ }^{10}$ More recently lenalidomide alone has been shown to delay progressions of HR-SMM; ${ }^{11}$ however, there was a high rate of treatment discontinuation and secondary cancers in the lenalidomide cohort. ${ }^{11}$ Ongoing 
clinical trials are also evaluating alternative treatment strategies to delay progression of HR-SMM. ${ }^{12}$ Most recently, next-generation sequencing (NGS) analysis of MGUSSMM-MM patients has proven to be a useful tool to decipher the timing and chronology of disease initiation events. ${ }^{13,14}$ In the near future, the combination of genomic signatures and markers of disease burden will likely enable identification of those SMM patients who may benefit from early intervention, and definition of the optimal time to initiate treatment to avoid the development of clinical sequelae. Assessment of the value of early intervention must balance the benefit of delaying/preventing symptomatic $\mathrm{MM}$ against the risk of adverse events, and such early interventions should be of finite duration.

\section{Prognostic factors and risk stratification}

Clinical and laboratory factors including disease stage, cytogenetic abnormalities, and depth of response to therapy can impact survival of MM patients. ${ }^{15}$ Cytogenetic analysis and fluorescence in situ hybridization (FISH)based genetic profiling should be routinely performed to evaluate disease biological behavior and prognosis. ${ }^{16}$ Among the poor prognostic markers, del(17p) and $t(4 ; 14)$ are the most informative ${ }^{17,18}$ concomitant secondary cytogenetic abnormalities may impact prognosis. ${ }^{4}$ The International Staging System (ISS), based on albumin and $\beta_{2}$-microglobulin levels, is most widely used, ${ }^{19}$ and has been revised (R-ISS) to incorporate lactate dehydrogenase $(\mathrm{LDH})$ and HR-FISH abnormalities. ${ }^{20}$ Given the genomic complexity of MM, more sophisticated techniques including gene expression profiling, mutational status, and copy number abnormalities have been used, alone or in combination with FISH-based approaches, to more deeply characterize disease biology and prognosis. For example, newly diagnosed MM (NDMM) patients carrying HR del(17p) may be further stratified using subclonal analysis. ${ }^{21}$ Targeted sequencing has been used as an alternative to whole exon sequencing to specifically analyze fractions of the genome and provide more accurate risk stratification. $^{22}$ Although not widely incorporated into clinical practice, these approaches will help to define future personalized treatment strategies in $\mathrm{MM}$.

\section{Assessment of response: minimal residual disease}

The high rate of complete response (CR) observed with the introduction of novel agents has led to the need for metrics capable of detecting even deeper responses to be developed. Response criteria are based on assessment of monoclonal protein in serum and urine, as well as BM evaluation. However, these parameters alone are not sensitive enough to detect low levels of residual tumor cells in the BM. ${ }^{23}$ More recently, both retrospective meta-analyses and prospective clinical trials have demonstrated the values of measuring minimal residual disease (MRD) within the $\mathrm{BM}$ using next-generation flow (NGF) or NGS, and at extramedullary sites using imaging such as PET/CT. ${ }^{24,25}$ The International Myeloma Working Group (IMWG) updated response criteria now include MRD status defined by absence of BM PC by NSG or NGF with a minimum sensitivity of 1 in $10^{5}$ nucleated cells in patients with $\mathrm{CR}$, providing guidelines that can be uniformly interpreted and applied in the context of clinical trials ${ }^{26} \mathrm{MRD}$ should be evaluated over the course of the disease, informing disease biology and treatment. ${ }^{26}$ For example, the DFCI/IFM clinical trial comparing lenalidomide-borte- zomib-dexamethasone followed by early versus late autologous stem cell transplant (ASCT) showed that MRD negativity at the level of $10^{-6}$ was associated with prolonged progression-free survival (PFS) and OS. ${ }^{27,28}$ Moreover, those patients with MRD-BM who were also imaging (PET/CT scan) MRD negativity had the best outcome. ${ }^{27,28}$ Whether MRD-negativity should represent the goal of therapy for all patients with NDMM or relapsed/refractory (RRMM), or whether treatment decisions should be predicated on $\mathrm{MRD}$ status, is still the focus of ongoing clinical trials.

\section{Biologically-based treatments}

High-dose chemotherapy plus ASCT remains the standard of care for NDMM patients of physiologic age 70 years or younger who have adequate cardiac, pulmonary, hepatic and renal function. ${ }^{4}$ Patients who are ineligible for transplant receive induction regimens dependent upon their frailty status. ${ }^{4}$ In both groups, the integration of scientifically-informed combinations of novel agents including immunomodulatory drugs (IMiD), proteasome inhibitor (PI), dexamethasone, and more recently monoclonal antibodies (mAbs), has transformed the treatment paradigm and patient outcome. However, genomic and clonal evolution in the tumor-promoting BM milieu underlies relapse of disease in most patients, and novel therapies are urgently needed.

\section{Direct targeting of multiple myeloma cell dependencies - Multiple myeloma "lineage" dependencies}

Tumor cells may crucially rely on survival mechanisms that are imprinted during lineage development, namely lineage-dependency. ${ }^{29}$ For example, the clinical success of first-in-class PI bortezomib in MM has validated the heightened dependency of MM cells on the protein quality control pathway as a therapeutic target..$^{30-32}$ The ubiquitin-proteasome system (UPS) is the primary mechanism for maintaining protein homeostasis. ${ }^{33}$ In normal PC, high protein turnover due to immunoglobulin production requires intact proteasome function, and this dependency is even higher in MM PC with aberrant protein turnover which further increases proteasome load. PI can overwhelm the imbalance between proteasome degradative capacity and proteasome load, ${ }^{3436}$ leading to endoplasmic reticulum stress due to accumulation of misfolded and unfolded proteins, activation of the unfolded protein response, and cell death. Since proteins involved in cell proliferation and apoptosis, cell-cycle, DNA repair, and metabolism are substrates of the proteasome, PI inhibitor bortezomib has broad effects. ${ }^{37,38}$ It triggers both intrinsic and extrinsic MM cell apoptosis and MM cell cycle arrest, and modifies bone turnover and osteoclast activity in the $\mathrm{BM}^{38}$ Bortezomib inhibits the NFKB pathway by blocking degradation of its inhibitor, IкB. ${ }^{33,38}$ Importantly, NFkB is a major oncogenic pathway in $\mathrm{MM}$, which mediates $\mathrm{MM}$ survival and DNA repair, promotes interactions of MM cells-BM accessory cells via the transcription of adhesion molecules, as well as modulating transcription of cytokines (such as IL-6, VEGF, IGF-1), which in turn mediate MM growth and drug resistance, and confer immunosuppression in the $\mathrm{BM}^{33,38}$

Over the disease course, MM cells acquire resistance to bortezomib via genetic and non-genetic mechanisms. ${ }^{33}$ Extensive preclinical research has delineated mechanisms 
of PI resistance and informed strategies to overcome resistance. Second-generation PI have been generated to overcome bortezomib resistance. The irreversible covalent epoxyketone PI carfilzomib, either alone or in combination with lenalidomide, has been approved to treat RRMM. $^{39}$ Ixazomib, the first oral boronic acid-based PI, has been approved, alone and in combination with lenalidomide, to treat RRMM; ${ }^{40}$ this all-oral regimen showed low toxicity profile and improved patient quality of life. The pan-PI marizomib, which penetrates the central nervous system (CNS), also demonstrates anti-MM activity in the setting of bortezomib resistance. ${ }^{41}$ Given the multitude of available PI, the side effect profiles and identification of biomarkers of PI resistance/sensitivity will determine their optimal and rational use.

Targeting upstream components of the ubiquitin proteasome system (UPS) has recently emerged as a promising strategy to overcome PI resistance. Therapeutic targeting of deubiquitylating enzymes (DUB) and the 19S proteasome-associated ubiquitin receptor Rpn13 overcame PI resistance in preclinical studies. ${ }^{42-45}$ However, the first-inhuman trial of USP14/UCHL5 DUB inhibitor for RRMM has been stopped due to dose-limiting toxicity. Targeted therapies against Rpn13 have been developed for evaluation in setting of PI resistance. ${ }^{46}$ An alternative approach to overcome PI resistance is the concomitant block of the aggresome/autophagy pathway using an inhibitor of histone deacetylase 6 (HDAC6), which is recruited to maintain proteostasis balance as an adaptive response mechanism. $^{38}$

Lineage vulnerabilities in $\mathrm{MM}$ also include aberrant transcription factor (TF) regulatory networks controlling lineage factor IRF4. ${ }^{47}$ Although direct targeting of TF represents an attractive strategy, there are no available inhibitors for clinical application. However, we found that aberrant regulatory KDM3A-IRF4-KLF2 loop may be effi-

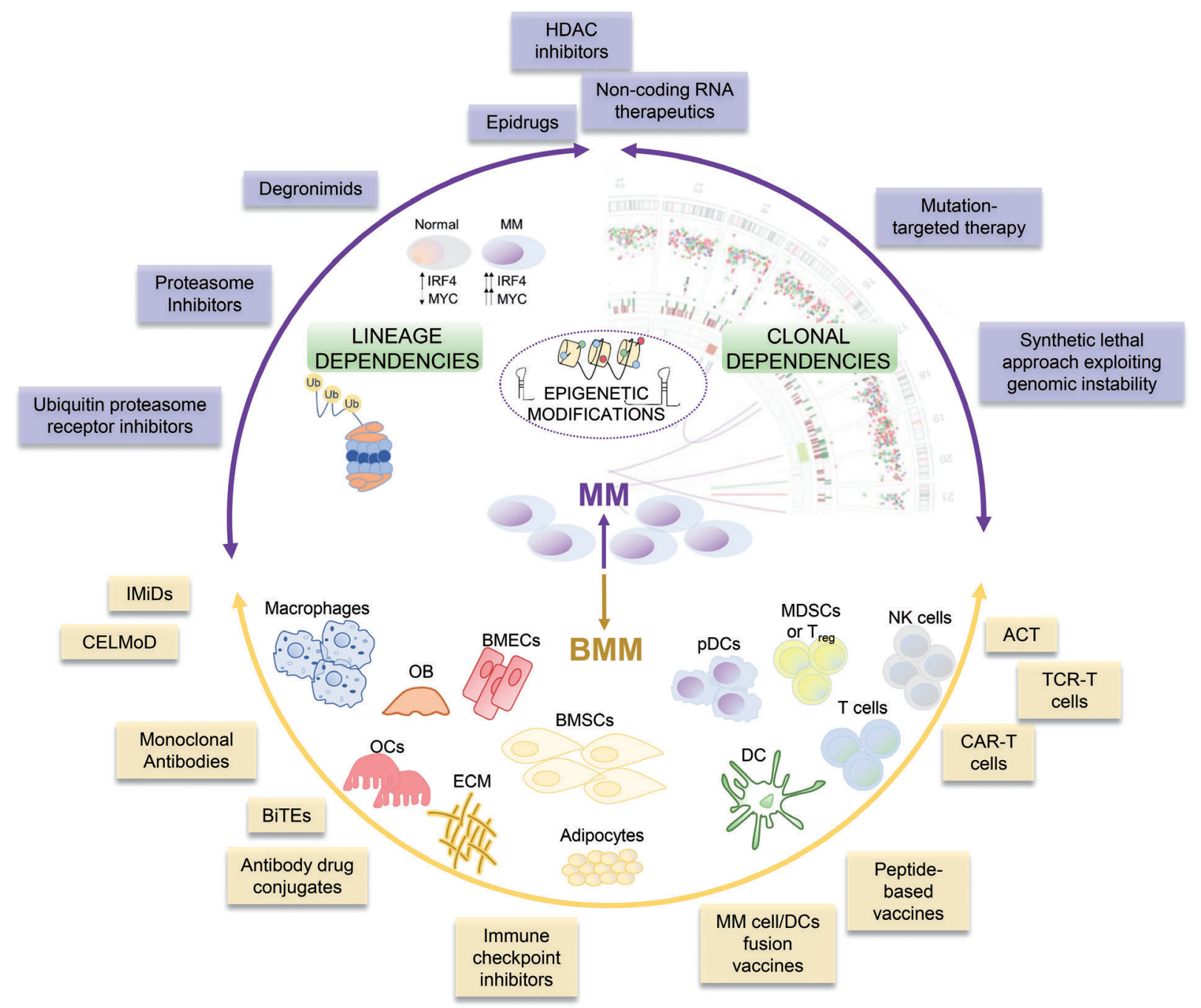

Figure 1. Overview of the different anti-multiple myeloma (MM) strategies discussed in the review. Purple: strategies designed to directly target MM cell vulnerabilities; we can distinguish those exploiting "lineage dependencies" or "clonal dependencies". (Center) Strategy targeting "epigenetic modifications" that may broadly affect both lineage and clonal vulnerabilities. (Bottom, yellow) Strategies aiming to disrupt MM-bone marrow microenvironment (BMM) interplay and restore host immunosurveillance. Purple double pointed arrows: MM-related approaches; yellow double pointed arrows: BMM-related approaches. These highlight the fact that one specific treatment, even in case of target therapy, may also affect multiple cellular components/interactions thus amplifying the therapeutic effects. OB: osteoblast; OC: osteoclasts; BMEC: bone marrow endothelial cells; ECM: extracellular matrix; BMSC: bone marrow stromal cells; DC: dendritic cell; pDC: plasmacytoid DC; MDSC: myeloid derived suppressor cells; Treg: regulatory T cell. 
ciently targeted by KDM3A inhibitor, which restores IRF4 and KLF2 promoter methylation and suppresses their transcription, thereby resulting in decreased $\mathrm{MM}$ cell homing to the BM and direct anti-MM toxicity. ${ }^{48}$ Moreover, repression of IRF4 transcription is observed after lenalidomide treatment, which triggers cereblon (CRBN)-mediated degradation of IRF4 transcriptional activator IKZF3..$^{49,50}$ From these findings, a new platform technology has been developed to trigger selective protein degradation. Specifically, degronimids, also known as proteolysis-targeting chimeras (PROTAC), are designed by conjugating the small-molecule binder of the target protein to an E3 ubiquitin ligase binding scaffold, such as the analogs of thalidomide which bind CRBN. ${ }^{51}$ This approach will allow for the therapeutic degradation of protein substrates that are otherwise challenging to target.

\section{- Multiple myeloma "clonal" dependencies}

Large inter-patient and intra-patient genetic heterogeneity limits the identification of universal drivers of MM. However, several oncogenic dependencies are primary events related to mutations in driver genes and primary translocations..$^{52}$ Translocations or gains of MYC locus (along with dysregulation of upstream signaling pathways, such as IRF4) support an oncogenic role of MYC in $\mathrm{MM}$, especially in the context of MGUS-MM transition. ${ }^{53}$ Alteration of the transcriptional program of MYC, and of its functional collaborators such as E2F1, promotes oncogenic signaling and PC survival..$^{54,55}$ Frequent gene mutations in MM include RAS, either KRAS or NRAS, with subsequent activation of the MAPK pathway, BRAF, DIS3, and FAM46C. ${ }^{56}$ Their role as prognostic factors has not been completely defined, as only TP53 mutation (6-8\% of patients at diagnosis) clearly confers worse patient outcome. ${ }^{57}$ Mutation-targeted treatments in MM are often compromised by intra-clonal heterogeneity. Specifically, deep sequencing has identified a complex subclonal structure in $\mathrm{MM}$ with different patterns of clonal evolution impacted by BM, immune response, and therapy. ${ }^{58}$ In this complex scenario, MM cells may share common mutations, but they may also express additional subclones which compromise mutation-targeting therapies. ${ }^{58,59}$ The MyDRUG trial is enrolling patients with relapsed MM based upon genomic sequencing; patients receive a specific treatment targeting their unique tumor mutations, along with standard-of-care treatment. ${ }^{60}$ This trial will reveal whether the abnormal clone can be targeted, and provide the rationale for further derived clinical trials of targeted therapies.

Genomic complexity in $\mathrm{MM}$ is due to genomic instability and ongoing DNA damage. ${ }^{61} \mathrm{MM}$ cells display hyperactivation of DNA repair mechanisms which confer a survival advantage and drug resistance with increasing numbers of new mutations over time. ${ }^{62}$ These aberrant processes may reveal new vulnerabilities. For example, in MM patients, in whom ongoing DNA damage occurs concurrently with low Hippo co-transcription factor (YAP1) levels, MM cell apoptosis is prevented. Conversely, inhibition of STK4 rescues YAP1 and triggers DNA-damageinduced apoptosis, providing the framework for clinical evaluation of STK4 inhibition. ${ }^{63}$ A second example is the induction of "BRCAness" status in MM cells by bortezomib, thereby increasing their sensitivity to PARP inhibitors. ${ }^{37}$ Finally, MYC amplification in MM can induce DNA response pathway and reactive oxygen species; the former can be blocked by ATR inhibitors and the latter can be increased by bortezomib, together triggering $\mathrm{MM}$ cell apoptosis in a synthetic lethal mechanism. ${ }^{61}$

\section{- Multiple myeloma epigenetic modifications}

Epigenetic alterations affect regulation of gene activity and expression, without altering gene sequence. Such alterations are associated with $\mathrm{MM}$ onset and progression, and modulate several important biological processes. ${ }^{64}$ Among epigenetic changes, DNA methylation, histone modification, and non-coding RNA (ncRNA) deregulation are the best characterized. ${ }^{64}$ Global hypomethylation of the genome characterizes the transition from MGUS to $\mathrm{MM}$, whereas pervasive genome re-methylation occurs in the transition from MM to more aggressive leukemic stage (PCL) ${ }^{64}$ Universal overexpression of histone methyltransferase MMSET is detected in patients carrying $t(4 ; 14)$ translocation and promotes MM cell survival by activating oncogenic MAPK pathway, increasing MYC and IRF4 transcription, and inducing chemo-resistance through enhancing DNA repair mechanisms. ${ }^{65,66}$ Therefore, development of MMSET inhibitor represents a promising therapeutic strategy for this subset of MM. We have demonstrated the oncogenic role and prognostic relevance of type II arginine methyltransferase PRMT5 in MM, whose inhibition results in $\mathrm{MM}$ cell killing via NFKB inhibition, thus providing the rationale for clinical trials targeting PRMT5 in MM. ${ }^{67}$

Histone deacetylases (HDAC) are generally hyperactive in $\mathrm{MM}$, and HDAC inhibitors are the most investigated epigenetic drugs. ${ }^{65}$ Preclinical studies have led to clinical trials and the approval of non-selective HDAC inhibitor panobinostat in combination with bortezomib in RRMM. ${ }^{68}$ However, increased toxicity observed with panobinostat prompted the development and translation of selective HDAC6 inhibitors (ricolinostat and ACY 241), which showed promising results and lower toxicity in combination with bortezomib and dexamethasone. ${ }^{69,70}$

Over the last decade, extensive studies have also highlighted the contribution of the ncRNA compartment in MM pathogenesis and progression. Specifically, microRNAs (miRNAs) are key regulators of gene expression at the post-transcriptional level, as they can induce either translational repression or degradation of target mRNAs upon total or partial complementary binding with $3^{\prime}$ untranslated region ( $3^{\prime}$ UTR) ${ }^{71}$ Given the multitude of targets for a single miRNA, these molecules harbor the potential to concomitantly regulate multiple biological processes. Preclinical data have defined their oncogenic (miR-221/222,-21,-17-92 cluster) or tumor suppressive (miR-29b,-34a,-125b,-15,-16) roles in MM associated with repression or overexpression, respectively, of genes involved in essential pro-survival pathways. ${ }^{72-77}$ The role of miRNAs has been similarly investigated in the context of drug resistance, BM-PC interaction, and bone disease. ${ }^{78-81}$ Although miRNA-based therapeutics have not yet translated into US Food and Drug Administration (FDA)approved drugs, several candidates are being tested in other diseases and will soon be evaluated in $\mathrm{MM}^{82}$ using miRNA replacement or inhibition strategies. As they are endogenous antisense of mRNAs, their replacement is likely to induce a "natural" effect on the targets, with less off-target effects compared to siRNAs; moreover, the recent availability of in vivo delivery systems now allows for clinical trials. ${ }^{83}$ Likewise, miRNA inhibition strategies take advantage of new antisense oligonucleotide tech- 
nologies, and ongoing early trials in several cancers will likely pave the way for their investigation in $M M^{83}$ Finally, long ncRNAs (IncRNA) represent major regulators of gene expression and chromatin dynamics by interacting with DNA and proteins. ${ }^{84}$ LncRNA genes outnumber protein-coding genes, with a partner of expression often restricted to specific cell types or conditions. ${ }^{84}$ With few exceptions, however, their functional role is still largely obscure in $M M{ }^{85}$ We recently described the IncRNA landscape in $\mathrm{MM}$, and their role as independent predictors of clinical outcome; ${ }^{86}$ ongoing and future studies will define their role in disease pathogenesis and as potential therapeutic targets.

\section{Targeting the tumor-bone marrow microenvironment interface}

Disrupting the interactions of MM cells with the BM represents an ideal therapeutic strategy in $\mathrm{MM}$, as shown by agents such as $\mathrm{IMiD}$ which remain active against $\mathrm{MM}$ even in the BM milieu. Cellular and non-cellular components of the BM niche support $\mathrm{MM}$ cell proliferation, migration, survival and drug resistance, while also conferring immunosuppression, and therefore represent targets for novel therapeutics. ${ }^{4,87,88}$

\section{- Immunomodulatory drugs}

Extensive preclinical and clinical studies have led to the FDA approval of the IMiD thalidomide and its more potent derivatives lenalidomide and pomalidomide for treatment of both NDMM and RRMM. ${ }^{89-92}$ IMiD induce direct cytotoxic effects on MM cells including growth arrest and caspase-8-mediated apoptosis, associated with CRBN-dependent degradation of IKZF1/3 followed by IRF4 downregulation. ${ }^{49,50,93}$ In the BM microenvironment they abrogate $\mathrm{MM}$ cell adhesion to the BM, modulate cytokine and growth factor secretion, inhibit angiogenesis, and most importantly, upregulate $\mathrm{T}, \mathrm{NK}$, and NKT cells, while downregulating regulatory $\mathrm{T}$ cells., ${ }^{3,93}$ Mechanistically, binding to CRBN has also been implicated in mediating the immune-related effects of IMiD, as IKZF1/3 degradation in T cells increases their secretion of cytokines including IL-2. ${ }^{94}$ This mechanism is also associated with increased natural killer (NK) and NK-T-cell cytotoxicity and antibody-dependent cellular cytotoxicity (ADCC) (i.e., anti-CD38, daratumumab and -CD20, rituximab) observed after IMiD treatment. ${ }^{95,96}$ Furthermore, IMiD can also enhance NK and T-cell cytotoxicity by triggering granzyme-B via either CRBN- or ZAP-70-dependent mechanisms. ${ }^{95}$ Preclinical and clinical studies have already confirmed the strong synergism of IMiD with PI and with $\mathrm{mAb} .{ }^{97-102}$ Recently, iberdomide, a higher affinity CRBN E3-ligase modulator (CELMoD), showed significant preclinical activity against IMiD-resistant $\mathrm{MM}$ cells, ${ }^{103}$ and ongoing clinical trials are examining its efficacy in RRMM resistant to lenalidomide and pomalidomide. Promising results have also been recently reported with the more potent CELMoD CC-92480, currently under investigation to treat IMiD-resistant RRMM. ${ }^{104}$

\section{- Immune-based therapies}

Loss of immune surveillance supports MM growth and resistance, and is associated with alterations in accessory and immune cells in the BM. ${ }^{105}$ Moreover, there is increasing evidence that evolving immune dysfunction is an important determinant of progression from MGUS/SMM to symptomatic MM. ${ }^{105}$ For example, functional interaction of plasmacytoid dendritic cells (DC) with MM cells promotes their survival and drug resistance, ${ }^{106}$ providing the framework for targeting this interaction in novel therapies. ${ }^{107-109}$ Similarly, myeloid derived suppressor cells, regulatory $\mathrm{T}$ cells, Th17 cells, tumor-associated macrophages, mesenchymal stromal cells, and osteoclasts significantly contribute to tumor immune escape and immunocompromised clinical status. ${ }^{110}$ Immune escape is also mediated, at least in part, by increased expression of immune checkpoints, i.e., PD-1/PD-L1, in T cells and MM cells, associated with disease progression from MGUS and SMM to MM. ${ }^{111}$ Although preclinical data have suggested the therapeutic utility of PD-1/PD-L1 blockade, early clinical trials have been discouraging. ${ }^{111}$ No singleagent activity of pembrolizumab has been shown, and importantly, two randomized clinical trials evaluating pembrolizumab in combination with IMiD to treat RRMM were closed due to excessive mortality. ${ }^{111}$ Ongoing studies are characterizing the role of the other immune checkpoint or agonist proteins (i.e., LAG 3 or TIGIT and OX40, respectively) as potential therapeutic targets, alone and in combination with MM targeted and immune therapies. ${ }^{112}$ Multiple $\mathrm{mAb}$ targeting MM surface antigens can trigger ADCC, antibody-dependent cellular phagocytosis, complement activation, and direct effect on MM cells. ${ }^{113}$ Elotuzumab and daratumumab target SLAMF7 and CD38, respectively. ${ }^{113}$ Elotuzumab also directly activates NK cells and is FDA approved in combination with lenalidomide or pomalidomide in RRMM.99,100 Daratumumab has shown remarkable extent and frequency of response, leading to its FDA approval as a single agent or in combination with IMiD and PI in both RRMM and NDMM..$^{97,98,114,115}$ The recent GRIFFIN trial compared standard lenalidomide-bortezomib-dexamethasone with or without daratumumab in transplant eligible patients, and showed deeper responses and MRD negativity rate in the daratumumab-treated patient cohort. ${ }^{116}$ Moreover, recent approval of subcutaneous formulation of daratumumab will dramatically reduce patient treatment times. ${ }^{117}$ More recently, a new CD38directed $\mathrm{mAb}$, isatuximab, has been approved in combination with pomalidomide-dexamethasone to treat RRMM; ${ }^{101}$ whether it is effective even in daratumumab refractory $\mathrm{MM}$ remains to be determined.

Monoclonal antibody technologies have also provided the framework for the development of Ab-drug conjugates $(\mathrm{ADC})$ and bispecific $\mathrm{T}$-cell engagers (BiTE). In the former, the conjugation with cytotoxic chemicals (such as auristatin) via synthetic linkers provides for direct tumor killing, and the $\mathrm{mAb}$ provides for selective tumor cell targeting, as well as immune-mediated cytotoxicity. ${ }^{118-122} \mathrm{~B}$ cell maturation antigen (BCMA)-directed ADC are currently under investigation in both preclinical and clinical settings, and represent a promising approach due to the highly specific expression on BCMA on MM cells and late memory B cells, as well as the role of the BCMA/APRIL pathway in supporting $\mathrm{MM}$ cell survival in the BM. ${ }^{113,118,123}$ The bi-specificity of the BiTE (mainly for CD3 on T cells and several $\mathrm{MM}$-associated antigens, such as BCMA and GPRC5D) allows for engagement of $\mathrm{T}$ cells with tumor cells, resulting in formation of cytolytic synapses and tumor lysis. ${ }^{124-126}$ Although results from early trials look promising, longer follow-up in larger studies are needed to assess the clinical benefit and potential toxicity. 
Importantly, BCMA ADC and BiTE have the advantage for "off the shelf" availability and universal use.

Cellular therapies represent an additional strategy to boost MM-specific immunity using either adoptive T-cell (ACT) or engineered T-cell approaches. ${ }^{3}$ Clinical experience with ACT using marrow-infiltrating lymphocytes (MIL) in MM has shown promise in achieving memory immune responses and stable disease. ${ }^{127}$ Importantly, advances in engineering technologies have allowed for both T-cell receptor (TCR) and chimeric antigen receptor (CAR) T-cell approaches. ${ }^{128}$ CAR are chimeric proteins that bring together the signaling moieties of TCR complexes and the variable domains of $\mathrm{Ab}$ which recognize a tumor-associated antigen. ${ }^{128,129}$ Co-stimulatory molecules have been included in the second-generation CAR-T cells to enhance T-cell activation by mimicking a physiological T-cell response. ${ }^{128,129}$ After genetic modification, a patient's T cells expressing the chimeric protein can be expanded ex vivo, and then activate a specific $\mathrm{T}$-cell response once reinfused to the patient. ${ }^{128,129}$ This allows CAR-T cells, in contrast to TCR-T cells, to recognize unprocessed tumor antigen in an MHC-independent manner. ${ }^{128,129} \mathrm{~A}$ major determinant of successful CAR-T therapy is the identification of a target uniquely and highly expressed by MM cells, thus limiting the occurrence of "off-target" effects. Among a variety of antigen targets, BCMA is the most frequently used due to its selectivity for normal plasma and MM cells. Several CAR-T products have been clinically tested in heavily pre-treated (PI-IMiD-CD38 mAb) RRMM, and have demonstrated remarkable deep (MRD ) responses. ${ }^{129-132}$ Clinical experience has also helped to improve management of the most commonly observed toxicities of CAR-T cells, including cytokine release syndrome and neurotoxicity. ${ }^{128,129}$ To date, however, most patients have relapsed, and ongoing research is assessing mechanisms of resistance to CAR-T, utilizing combination immune approaches with CAR-T, and using CAR-T earlier in the disease course in order to achieve more durable responses.

Lastly, vaccination strategies have been developed to improve antigen-specific memory anti-MM immunity. Specifically, multi-peptide-based vaccines induce effective and durable memory peptide-specific CTL in SMM patients, providing the rationale for their clinical evaluation to delay progression from SMM to active disease. ${ }^{133,134}$ More recently, a novel engineered heteroclitic BCMA peptide has been used to induce a BCMA-specific memory anti-MM immunity, suggesting its potential use in vaccination and/or ACT strategies to generate longlasting immunity against $\mathrm{MM} .{ }^{135}$ As alternative vaccination strategy employs MM cell/DC fusion vaccines to generate anti-MM immunity in the post-ASCT setting, this vaccine induces anti-MM immunity and enhances depth of response. ${ }^{136}$ The most significant obstacle to successful vaccination therapy in $\mathrm{MM}$ is the disease- and treatment-related immune dysfunction, which may limit the immune responses in vivo. As such, a randomized trial comparing lenalidomide versus lenalidomide plus MM cell/DC fusion vaccine post-transplant is ongoing (clinicaltrials.gov identifier: NCT02728102) to determine whether combination of vaccination with IMiD may improve its efficacy. Overall, future treatment approaches will likely rely on the optimal combination of targeted and immunebased strategies to obtain a durable anti-MM response and restore the host immune-surveillance.

\section{Future directions}

Despite tremendous advances, the clinical management of $\mathrm{MM}$ patients remains challenging, since acquisition of resistance underlies relapse of disease in most patients. Correlative science studies on patient samples are delineating mechanisms of resistance to both targeted and immune agents in order to inform clinical strategies to overcome resistance and improve patient outcome. Development of second-generation more potent drugs of the same class has overcome both PI and IMiD resistance, as have combination therapies with agents targeting pathways mediating resistance. Identification of biomarkers of patient MM resistance/sensitivity may further inform sequential and combination therapies in the future. Importantly, agents targeting novel MM vulnerabilities are urgently needed. For example, selinexor, a selective inhibitor of nuclear export of tumor suppressor proteins, growth factors, and mRNAs of oncogenic proteins, has recently been approved in triple-class (PI-IMiD-anti-CD38 $\mathrm{mAb}$ ) refractory $\mathrm{MM}^{137}$

A similar scenario of resistance is now beginning to appear for immune-based approaches, with several possible explanations. Loss of targeted antigens (such as $\mathrm{BCMA}, \mathrm{CD} 38$ ) is a common event, either due to loss with tumor evolution or to suppression in the face of immune pressure. Multi-antigen targeting may potentially overcome this obstacle, and several trials are evaluating this strategy. ${ }^{129}$ Similarly, circulating antigen in a soluble form may potentially interfere with immune-targeted approaches. For example, high levels of soluble BCMA have been detected in MM patients, and anti-BCMA CAR-T therapy is being combined with $\gamma$-secretase inhibitor to block BCMA cleavage from the MM cell surface (clinicaltrials.gov identifier: NCT03502577). Additional resistance may be intrinsic to the technology or modality, and ongoing efforts to increase CAR-T cell expansion and persistence in vivo include enriching for early memory $\mathrm{T}$ cell phenotype, optimizing CAR design to avoid antigenindependent tonic signaling, and/or intensifying lymphodepletion to promote CAR-T cell persistence. ${ }^{129}$

Lastly, T-cell exhaustion and the immunosuppressive BM may contribute to both targeted- and immune-therapy resistance. ${ }^{128}$ Restoration of host anti-MM immunity represents an important unmet need in MM. Several models, such as SCID-hu and SCID-synth-hu, have been developed to recapitulate the in vivo growth on patient MM cells in the context of BM. ${ }^{138,139}$ However, understanding the role of the immune system in disease pathobiology requires the use of immunocompetent models (such as the $5 \mathrm{~T}$ and $\left.\mathrm{Vk}^{*} \mathrm{MYC}\right){ }^{53,140}$ This is critical for evaluating not only immune therapies, but also targeted MM agents, such as bortezomib, which induce immunogenic cell death in vivo.

A recent area of investigation in $\mathrm{MM}$ is assessing the role of gut microbiome in shaping the immune system response, including anti-tumor immunity. For example, a commensal bacterium Prevotella heparinolytica promotes progression of $\mathrm{MM}$ by favoring differentiation of Th17 cells in the gut, which migrate to the BM of Vk*MYC mice and activate eosinophils; targeting IL-17-eosinophil immune axis may, therefore, represent a potential treatment for HR-SMM. ${ }^{141}$ Abundance of Eubacterium limosum bacteria in the intestinal flora has been associated with relapse after allogeneic stem cell transplantation. ${ }^{142}$ 
Similarly, high presence of Eubacterium hallii in the intestinal microbiota correlates with achievement of MRD negativity. ${ }^{143}$ Although still in an early stage, studies of the MM microbiome may identify future biomarkers or therapeutic agents to improve MM patient outcome.

Finally, identification of specific biomarkers predictive of therapy response within a patient's heterogeneous MM has been a major focus of research. The first example of biomarker-driven anti-MM treatment in $\mathrm{MM}$ is the $\mathrm{Bcl}-2$ inhibitor venetoclax, whose safety and efficacy are predicated upon occurrence of $\mathrm{t}(11 ; 14)$ or presence of high levels of BCL2. ${ }^{144}$ Several trials in RRMM are showing efficacy of venetoclax, as monotherapy and in combination, restricted to this patient subset. ${ }^{145,146}$ Integration of current and future technologies may further guide disease management and allow for precision medicine. For example, encouraging results have recently been reported in a trial using a multi-omics approach integrating DNA and RNA sequencing to inform drug treatment for RRMM. ${ }^{147}$ The ongoing MyDRUG trial profiles relapsed MM, and is then examining whether targeting genomic abnormalities in combination with standard relapse MM therapy can delete the abnormal MM clone. ${ }^{60}$ This and other trials will inform the utility of precision medicine in $\mathrm{MM}$, especially in the presence of concomitant genetic abnormalities.

Given the multiple available treatment options, welldesigned randomized clinical trials are necessary to assess the superior efficacy of specific regimens with head-tohead comparison. For example, interim analysis of the phase III ENDURANCE trial did not show superior PFS of carfilzomib versus bortezomib in combination with lenalidomide-dexamethasone for NDMM. Importantly, regulatory randomized trial results require real-world validation, since patient age, frailty status, and comorbidity frequently do not reflect trial patients. High-dose melphalan with ASCT remains a standard of care, and its role in the era of novel therapies is under evaluation in the IFM/DFCI 2009 DETERMINATION and FORTE clinical trials. However, the recent use of quadruplet therapies including daratumumab in the CASSIOPEIA and GRIFFIN trials shows that the addition of $\mathrm{mAb}$ can achieve increased extent and depth of response to induction ther- apy, ${ }^{116,148}$ and whether high-dose melphalan and ASCT improves outcome of quadruplet therapy remains to be determined. Nonetheless, research continues to improve alkylating agents as well. For example, melflufen is a prodrug which is digested to melphalan by high levels of aminopeptidase in MM cells, thereby improving its therapeutic index. ${ }^{149,150}$ Ultimately, the future use of novel targeted and immune therapies, as well as the role of conventional therapies, will be defined by vulnerabilities within individual patients and/or patient subgroups.

\section{Conclusions}

Over the past decades, a deeper understanding of the complex MM pathobiology has informed drug development and clinical practice, resulting in significant improvements in patient outcome. Combination approaches targeting $\mathrm{MM}$ cells, disrupting $\mathrm{MM}$ cell/BM interactions, and enhancing anti-MM immune responses, have remarkably improved response extent and frequency. Remaining obstacles to cure include constitutive and evolving genomic heterogeneity in MM cells, as well as the immunosuppressive BM milieu. In the future, integration of advanced sequencing technologies profiling both the MM cell and BM accessory/immune cells will identify novel targets and inform more potent, selective, and well tolerated targeted and immune therapies. Long-term disease-free survival and potential cure in MM will require both achieving MRD negativity and restoring host anti$\mathrm{MM}$ immunity. Such patients can then be free of disease while off all therapies.

\section{Funding}

This work is supported by NIH/NCI grants SPOREP50CA100707 (KCA), R01-CA050947 (KCA), R01CA207237 (KCA), P01CA155258 (KCA) and R01CA178264 (KCA); and the Sheldon and Miriam Medical Research Foundation (KCA). KCA is an American Cancer Society Clinical Research Professor. AG is a Fellow of The Leukemia \& Lymphoma Society and a Scholar of the American Society of Hematology (ASH).

\section{References}

1. National Cancer Institute. Cancer stat facts: myeloma. Available from: https://seer.cancer.gov/statfacts/html/mulmy.html. Accessed 20 July 2020.

2. Cowan AJ, Allen C, Barac A, et al. Global burden of multiple myeloma: a systematic analysis for the Global Burden of Disease Study 2016. JAMA Oncol. 2018;4(9):1221-1227.

3. Anderson KC. Progress and paradigms in multiple myeloma. Clin Cancer Res. 2016;22(22):5419-5427.

4. Kumar SK, Rajkumar V, Kyle RA, et al. Multiple myeloma. Nat Rev Dis Primers. 2017;3:17046.

5. Rajkumar SV, Dimopoulos MA, Palumbo A, et al. International Myeloma Working Group updated criteria for the diagnosis of multiple myeloma. Lancet Oncol. 2014;15 (12):e538-548.

6. Mateos MV, Hernandez MT, Giraldo P, et al. Lenalidomide plus dexamethasone for highrisk smoldering multiple myeloma. $\mathrm{N}$ Engl J
Med 2013:369(5):438-447

7. Kyle RA, Greipp PR. Smoldering multiple myeloma. N Engl J Med. 1980;302(24):13471349

8. Kapoor P, Rajkumar SV. Smoldering multiple myeloma: to treat or not to treat. Cancer J. 2019;25(1):65-71.

9. Lakshman A, Rajkumar SV, Buadi FK, et al. Risk stratification of smoldering multiple myeloma incorporating revised IMWG diagnostic criteria. Blood Cancer J. 2018;8(6):59.

10. Mateos MV, Hernandez MT, Giraldo P, et al. Lenalidomide plus dexamethasone versus observation in patients with high-risk smouldering multiple myeloma (QuiRedex): long-term follow-up of a randomised, controlled, phase 3 trial. Lancet Oncol. 2016:17(8):1127-1136.

11. Lonial S, Jacobus S, Fonseca $R$, et al. Randomized trial of lenalidomide versus observation in smoldering multiple myeloma. I Clin Oncol. 2020;38(11):1126-1137.

12. D'Agostino M, Bertamini L, Oliva S, Boccadoro M, Gay F. Pursuing a curative approach in multiple myeloma: a review of new therapeutic strategies. Cancers (Basel). 2019;11(12).

13. Rustad EH, Yellapantula V, Leongamornlert $\mathrm{D}$, et al. Timing the initiation of multiple myeloma. Nat Commun. 2020;11(1):1917.

14. Aktas Samur A, Minvielle S, Shammas M, et al. Deciphering the chronology of copy number alterations in multiple myeloma. Blood Cancer J. 2019;9(4):39.

15. Rajkumar SV. Multiple myeloma: 2016 update on diagnosis, risk-stratification, and management. Am J Hematol. 2016;91(7): 719-734.

16. Sonneveld P, Avet-Loiseau H, Lonial S, et al. Treatment of multiple myeloma with highrisk cytogenetics: a consensus of the International Myeloma Working Group. Blood. 2016;127(24):2955-2962.

17. Avet-Loiseau H, Hulin C, Campion L, et al. Chromosomal abnormalities are major prognostic factors in elderly patients with multiple myeloma: the Intergroupe Francophone du Myelome experience. J Clin Oncol. 2013;31(22):2806-2809.

18. Kumar S, Fonseca R, Ketterling RP, et al. 

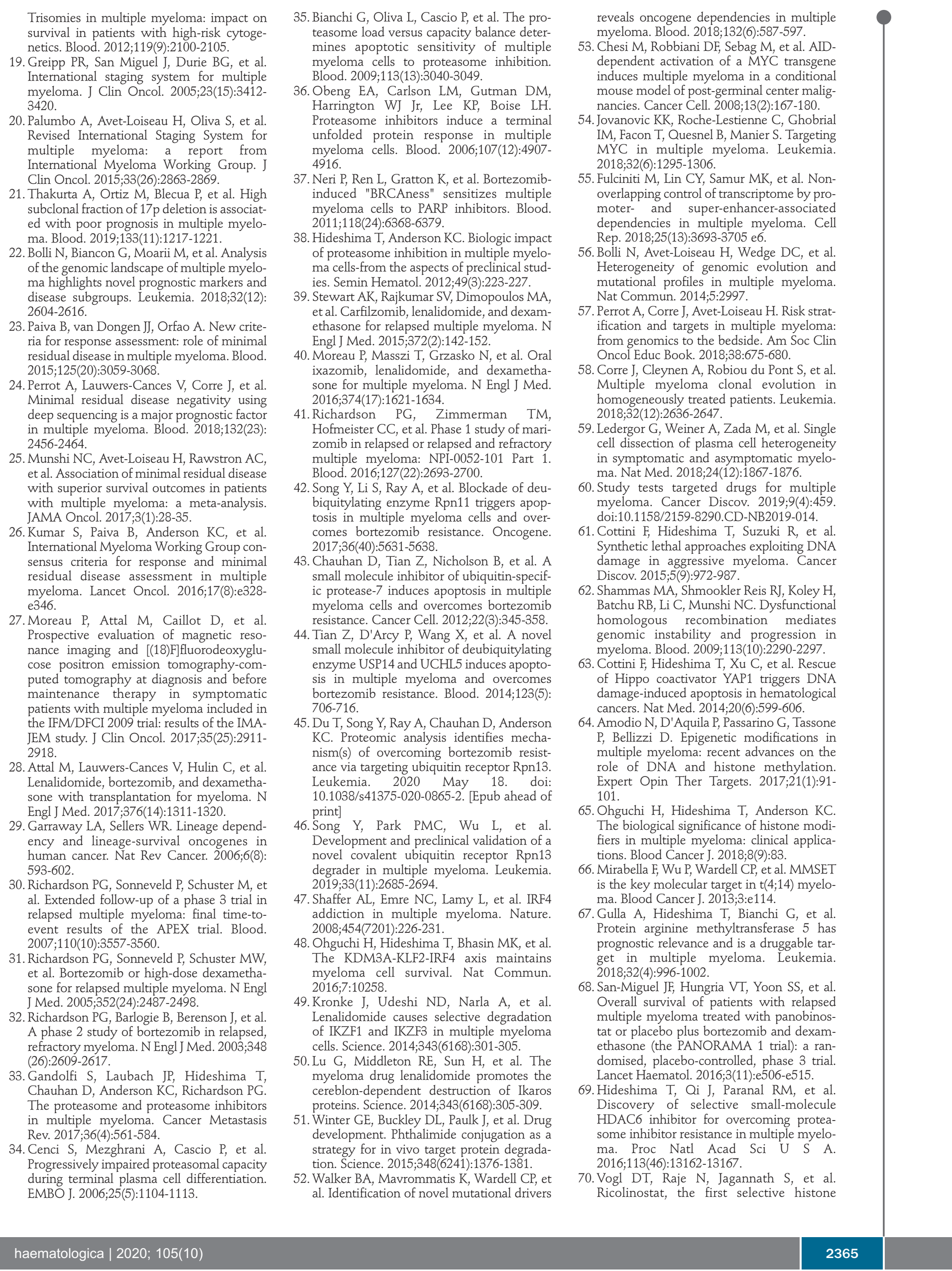
deacetylase 6 inhibitor, in combination with bortezomib and dexamethasone for relapsed or refractory multiple myeloma. Clin Cancer Res. 2017;23(13):3307-3315.

71. Bartel DP. MicroRNAs: genomics, biogenesis, mechanism, and function. Cell. 2004;116 (2):281-297.

72. Leone E, Morelli E, Di Martino MT, et al. Targeting miR-21 inhibits in vitro and in vivo multiple myeloma cell growth. Clin Cancer Res. 2013;19(8):2096-2106.

73. Morelli E, Biamonte L, Federico C, et al. Therapeutic vulnerability of multiple myeloma to MIR17PTi, a first-in-class inhibitor of pri-miR-17-92. Blood. 2018;132(10):10501063.

74. Amodio N, Stamato MA, Gulla AM, et al. Therapeutic targeting of miR-29b/HDAC4 epigenetic loop in multiple myeloma. Mol Cancer Ther. 2016;15(6):1364-1375

75. Di Martino MT, Leone E, Amodio N, et al. Synthetic miR-34a mimics as a novel therapeutic agent for multiple myeloma: in vitro and in vivo evidence. Clin Cancer Res. 2012;18(22):6260-6270.

76. Morelli E, Leone E, Cantafio ME, et al. Selective targeting of IRF4 by synthetic microRNA-125b-5p mimics induces antimultiple myeloma activity in vitro and in vivo. Leukemia. 2015;29(11):2173-2183

77. Caracciolo D, Di Martino MT, Amodio N, et al. miR-22 suppresses DNA ligase III addiction in multiple myeloma. Leukemia. 2019:33(2):487-498.

78. Gulla A, Di Martino MT, Gallo Cantafio ME, et al. A 13 mer LNA-i-miR-221 inhibitor restores drug sensitivity in melphalan-refractory multiple myeloma cells. Clin Cancer Res. 2016;22(5):1222-1233.

79. Zhao JJ, Chu ZB, Hu Y, et al. Targeting the miR-221-222/PUMA/BAK/BAX pathway abrogates dexamethasone resistance in multiple myeloma. Cancer Res. 2015;75(20): 4384-4397.

80. Botta C, Cuce M, Pitari MR, et al. MiR-29b antagonizes the pro-inflammatory tumorpromoting activity of multiple myelomaeducated dendritic cells. Leukemia. 2018:32(4):1003-1015.

81. Pitari MR, Rossi M, Amodio N, et al. Inhibition of miR-21 restores RANKL/OPG ratio in multiple myeloma-derived bone marrow stromal cells and impairs the resorbing activity of mature osteoclasts. Oncotarget. 2015;6(29):27343-27358.

82. Hong DS, Kang YK, Borad M, et al. Phase 1 study of MRX34, a liposomal miR-34a mimic, in patients with advanced solid tumours. Br J Cancer. 2020;122(11):16301637.

83. Rupaimoole R, Slack FJ. MicroRNA therapeutics: towards a new era for the management of cancer and other diseases. Nat Rev Drug Discov. 2017;16(3):203-222.

84. Morelli E, Gulla A, Rocca R, et al. The noncoding RNA landscape of plasma cell dyscrasias. Cancers (Basel). 2020;12(2).

85. Amodio N, Stamato MA, Juli G, et al. Drugging the lncRNA MALAT1 via LNA gapmeR ASO inhibits gene expression of proteasome subunits and triggers anti-multiple myeloma activity. Leukemia. 2018;32(9): 1948-1957.

86. Samur MK, Minvielle S, Gulla A, et al. Long intergenic non-coding RNAs have an independent impact on survival in multiple myeloma. Leukemia. 2018;32(12):2626-2635.

87. Chesi M, Mirza NN, Garbitt VM, et al. IAP antagonists induce anti-tumor immunity in multiple myeloma. Nat Med. 2016;22(12): 1411-1420.
88. Fan F, Bashari MH, Morelli E, et al. The AP1 transcription factor JunB is essential for multiple myeloma cell proliferation and drug resistance in the bone marrow microenvironment. Leukemia. 2017;31(7): 1570-1581.

89. Dimopoulos M, Spencer A, Attal M, et al Lenalidomide plus dexamethasone for relapsed or refractory multiple myeloma. $\mathrm{N}$ Engl J Med. 2007:357(21):2123-2132.

90. McCarthy PL, Owzar K, Hofmeister CC, et al. Lenalidomide after stem-cell transplantation for multiple myeloma. N Engl J Med. 2012;366(19):1770-1781.

91. Benboubker L, Dimopoulos MA, Dispenzieri A, et al. Lenalidomide and dexamethasone in transplant-ineligible patients with myeloma. N Engl J Med. 2014;371 (10):906-917.

92. Miguel JS, Weisel K, Moreau P, et al. Pomalidomide plus low-dose dexamethasone versus high-dose dexamethasone alone for patients with relapsed and refractory multiple myeloma (MM-003): a randomised, open-label, phase 3 trial. Lancet Oncol. 2013;14(11):1055-1066.

93. Davies F, Baz R. Lenalidomide mode of action: linking bench and clinical findings. Blood Rev. 2010;24(Suppl 1):S13-S19.

94. Gandhi AK, Kang J, Havens CG, et al. Immunomodulatory agents lenalidomide and pomalidomide co-stimulate $T$ cells by inducing degradation of $\mathrm{T}$ cell repressors Ikaros and Aiolos via modulation of the E3 ubiquitin ligase complex CRL4(CRBN). Br J Haematol. 2014;164(6):811-821

95. Hideshima T, Ogiya D, Liu J, et al. Immunomodulatory drugs activate $\mathrm{NK}$ cells via both Zap-70 and cereblon-dependent pathways. Leukemia. 2020 Apr 1. doi.org/10.1038/s41375-020-0809-x. [Epub ahead of print]

96. Luptakova K, Rosenblatt J, Glotzbecker B, et al. Lenalidomide enhances anti-myeloma cellular immunity. Cancer Immunol Immunother. 2013;62(1):39-49.

97. Facon T, Kumar S, Plesner $T$, et al. Daratumumab plus lenalidomide and dexamethasone for untreated myeloma. N Engl J Med. 2019;380(22):2104-2115

98. Dimopoulos MA, Oriol A, Nahi H, et al. Daratumumab, lenalidomide, and dexamethasone for multiple myeloma. $\mathrm{N}$ Engl Med. 2016;375(14):1319-1331.

99. Dimopoulos MA, Dytfeld D, Grosicki S, et al. Elotuzumab plus pomalidomide and dexamethasone for multiple myeloma. $\mathrm{N}$ Engl $\mathrm{J}$ Med. 2018;379(19):1811-1822.

100. Lonial S, Dimopoulos M, Palumbo A, et al. Elotuzumab therapy for relapsed or refractory multiple myeloma. N Engl J Med. 2015;373(7):621-631

101. Attal M, Richardson PG, Rajkumar SV, et al. Isatuximab plus pomalidomide and lowdose dexamethasone versus pomalidomide and low-dose dexamethasone in patients with relapsed and refractory multiple myeloma (ICARIA-MM): a randomised, multicentre, open-label, phase 3 study. Lancet. 2019;394(10214):2096-2107.

102. Martin T, Baz R, Benson DM, et al. A phase $1 \mathrm{~b}$ study of isatuximab plus lenalidomide and dexamethasone for relapsed/refractory multiple myeloma. Blood. 2017;129(25): 3294-3303.

103. Bjorklund CC, Kang J, Amatangelo M, et al. Iberdomide (CC-220) is a potent cereblon E3 ligase modulator with antitumor and immunostimulatory activities in lenalidomide- and pomalidomide-resistant multiple myeloma cells with dysregulated CRBN
Leukemia. 2020;34(4):1197-1201.

104. Hansen JD, Correa M, Nagy MA, et al. Discovery of CRBN E3 ligase modulator CC-92480 for the treatment of relapsed and refractory multiple myeloma. J Med Chem. 2020;63(13):6648-6676.

105. Zavidij O, Haradhvala NJ, Mouhieddine TH, et al. Ghobrial single-cell RNA sequencing reveals compromised immune microenvironment in precursor stages of multiple myeloma. Nature Cancer. 2020 Apr 27. doi.org/10.1038/s43018-020-0053-3. [Epub ahead of print]

106. Chauhan D, Singh AV, Brahmandam M, et al. Functional interaction of plasmacytoid dendritic cells with multiple myeloma cells: a therapeutic target. Cancer Cell. 2009;16(4): 309-323.

107. Ray A, Das DS, Song Y, et al. A novel agent SL-401 induces anti-myeloma activity by targeting plasmacytoid dendritic cells, osteoclastogenesis and cancer stem-like cells. Leukemia. 2017;31(12):2652-2660.

108. Ray A, Tian Z, Das DS, et al. A novel TLR-9 agonist C792 inhibits plasmacytoid dendritic cell-induced myeloma cell growth and enhance cytotoxicity of bortezomib Leukemia. 2014;28(8):1716-1724.

109. Ray A, Song Y, Chauhan D, Anderson KC. Blockade of ubiquitin receptor Rpn13 in plasmacytoid dendritic cells triggers antimyeloma immunity. Blood Cancer J. 2019;9(8):64.

110. Holthof LC, Mutis T. Challenges for immunotherapy in multiple myeloma: bone marrow microenvironment-mediated immune suppression and immune resistance. Cancers (Basel). 2020;12(4).

111. Costello C. The future of checkpoint inhibition in multiple myeloma? Lancet Haematol. 2019;6(9):e439-e440.

112. Costa F, Das R, Kini Bailur J, Dhodapkar K, Dhodapkar MV. Checkpoint inhibition in myeloma: opportunities and challenges. Front Immunol. 2018;9:2204.

113. Wudhikarn K, Wills B, Lesokhin AM Monoclonal antibodies in multiple myeloma: current and emerging targets and mechanisms of action. Best Pract Res Clin Haematol. 2020;33(1):101143

114. Chari A, Martinez-Lopez J, Mateos MV, et al. Daratumumab plus carfilzomib and dexamethasone in patients with relapsed or refractory multiple myeloma. Blood. 2019;134(5):421-431.

115. Spencer A, Lentzsch S, Weisel K, et al Daratumumab plus bortezomib and dexamethasone versus bortezomib and dexamethasone in relapsed or refractory multiple myeloma: updated analysis of CASTOR. Haematologica. 2018;103(12):2079-2087.

116. Voorhees PM, Kaufman JL, Laubach JP, et al Daratumumab, lenalidomide, bortezomib, and dexamethasone for transplant-eligible newly diagnosed multiple myeloma: GRIFFIN. Blood. 2020 Apr 23. doi 10.1182/blood.2020005288. [Epub ahead of print]

117. Mateos MV, Nahi H, Legiec W, et al Subcutaneous versus intravenous daratumumab in patients with relapsed or refractory multiple myeloma (COLUMBA): a multicentre, open-label, non-inferiority, randomised, phase 3 trial. Lancet Haematol. 2020;7(5):e370-e380.

118. Cho SF, Anderson KC, Tai YT. Targeting B cell maturation antigen (BCMA) in multiple myeloma: potential uses of BCMAbased immunotherapy. Front Immunol. 2018;9:1821.

119. Xing L, Lin L, Yu T, et al. A novel BCMA 
MM: current therapy and future prospects

PBD-ADC with ATM/ATR/WEE1 inhibitors or bortezomib induce synergistic lethality in multiple myeloma. Leukemia. 2020 Feb 14. doit: 10.1038/s41375-020-0745-9. [Epub ahead of print]

120. Trudel S, Lendvai N, Popat R, et al. Targeting B-cell maturation antigen with GSK2857916 antibody-drug conjugate in relapsed or refractory multiple myeloma (BMA117159): a dose escalation and expansion phase 1 trial. Lancet Oncol. 2018;19(12):1641-1653.

121. Kinneer K, Meekin J, Tiberghien AC, et al. SLC46A3 as a potential predictive bismarker for antibody-drug conjugates bearing noncleavable linked maytansinoid and pyrrolobenzodiazepine warheads. Chin Cancer Res. 2018;24(24):6570-6582.

122. Tai YT, Mays PA, Acharya C, et al. Novel anti-B-cell maturation antigen antibodydrug conjugate (GSK2857916) selectively induces killing of multiple myeloma. Blood. 2014;123(20):3128-3138.

123. Tai YT, Acharya C, An G, et al. APRIL and BCMA promote human multiple myeloma growth and immunosuppression in the bone marrow microenvironment. Blood. 2016; 127(25):3225-3236.

124. Frerichs KA, Broekmans MEC, Marin Soto JA, et al. Preclinical activity of JNJ-7957, a novel BCMAxCD3 bispecific antibody for the treatment of multiple myeloma, is potentiated by daratumumab. Chin Cancer Res. 2020;26(9):2203-2215.

125. Seckinger A, Delgado JA, Mover S, et al. Target expression, generation, preclinical activity, and pharmacokinetics of the BCMA-T cell bispecific antibody EM801 for multiple myeloma treatment. Cancer Cell. 2017;31(3):396-410.

126. Hip S, Tai YT, Blanset D, et al. A novel BCMA/CD3 bispecific T-cell engager for the treatment of multiple myeloma induces selective lysis in vito and in vino. Leukemia. 2017;31(8):1743-1751.

127. Noonan KA, Huff CA, Davis J, et al. Adoptive transfer of activated marrow-infilrating lymphocytes induces measurable antitumor immunity in the bone marrow in multiple myeloma. Sci Transl Med. 2015;7 (288):288ra78.

128. Rodriguez-Otero P, Paiva B, Engelhardt M, Prosper F, San Miguel JF. Is immunotherapy here to stay in multiple myeloma? Haematological. 2017;102(3):423-432.

129. D'Agostino M, Raje N. Anti-BCMA CAR Tcell therapy in multiple myeloma: can we do better? Leukemia. 2020;34(1):21-34.

130. Yarn Z, Gao J, Chen H, et al. A combination of humanised anti-CD19 and anti-BCMA CAR T cells in patients with relapsed or refractory multiple myeloma: a single-arm, phase 2 trial. Lancet Haematol. 2019;6(10):e521-e529.

131. Raje N, Berdeja J, Lin Y, et al. Anti-BCMA CAR T-cell therapy bb2121 in relapsed or refractory multiple myeloma. $\mathrm{N}$ Engl J Med. 2019;380(18):1726-1737.

132. Xu J, Chen LJ, Yang SS, et al. Exploratory trial of a biepitopic CAR T-targeting B cell maturation antigen in relapsed/refractory multiple myeloma. Proc Natl Acad Sci U S A. 2019;116(19):9543-9551.

133. Brae J, Smith R, Daley J, et al. Myeloma-specific multiple peptides able to generate cytotoxic T lymphocytes: a potential therapeutic application in multiple myeloma and other plasma cell disorders. Chin Cancer Res. 2012;18(17):4850-4860.

134. Brae J, Prabhala R, Voskertchian A, et al. A multiepitope of XBP1, CD138 and CS1 peptides induces myeloma-specific cytotoxic T lymphocytes in T cells of smoldering myeloma patients. Leukemia. 2015; 29(1):218-229

135. Bay J, Samur M, Richardson P, Munshi NC, Anderson KC. Selective targeting of multiple myeloma by $\mathrm{B}$ cell maturation antigen (BCMA)-specific central memory CD8(+) cytotoxic $\mathrm{T}$ lymphocytes: immunotherapectic application in vaccination and adofive immunotherapy. Leukemia. 2019; 33(9):2208-2226.

136. Rosenblatt J, Avivi I, Vair B, et al. Vaccination with dendritic cell/tumor fusions following autologous stem cell transplant induces immunologic and clinical responses in multiple myeloma patients. Chin Cancer Res. 2013;19(13):3640-3648.

137. Chari A, Vol DT, Gavriatopoulou M, et al. Oral selinexor-dexamethasone for tripleclass refractory multiple myeloma. $\mathrm{N}$ Engl J Med. 2019;381(8):727-738.

138. Tassone P, Keri P, Carrasco DR, et al. A chinically relevant SCID-hu in vive model of human multiple myeloma. Blood. 2005;106 (2):713-716.

139. Calimeri T, Battista E, Conforti F, et al. A unique three-dimensional SCID-polymeric scaffold (SCID-synth-hu) model for in vino expansion of human primary multiple myeloma cells. Leukemia. 2011;25(4):707711.
140. Rad J, Punt YA, van den Enden-Vieveen $\mathrm{MH}$, et al. The 5T mouse multiple myeloma model: absence of c-myc oncogene rearrangement in early transplant generaions. Br J Cancer. 1990;61(2):276-278.

141. Calcinotto A, Brevi A, Chess M, et al. Microbiota-driven interleukin-17-producing cells and eosinophils synergize to accelerate multiple myeloma progression. Nat Commune. 2018;9(1):4832.

142. Pele JU, Devin SM, Staffas A, et al. Intestinal microbiota and relapse after hematopoietic-cell transplantation. J Chin Oncol. 2017;35(15):1650-1659.

143. Pianko MJ, Devin SM, Littmann ER, et al. Minimal residual disease negativity in multipe myeloma is associated with intestinal microbiota composition. Blood Adv. 2019;3(13):2040-2044.

144. Touzeau C, Macias P, Amiot M, Moreau P. Targeting Bcl-2 for the treatment of multiple myeloma. Leukemia. 2018;32(9):18991907.

145. Kumar S, Kaufman JL, Gasparetto C, et al. Efficacy of venetoclax as targeted therapy for relapsed/refractory $t(11 ; 14)$ multiple myeloma. Blood. 2017;130(22):2401-2409.

146. Moreau P, Chanan-Khan A, Roberts AW, et al. Promising efficacy and acceptable safety of venetoclax plus bortezomib and examethasone in relapsed/refractory MM. Blood. 2017;130(22):2392-2400.

147. Lagena A, Bueno I, Melnekoff D, et al. Precision medicine for relapsed multiple myeloma on the basis of an integrative pultiomics approach. JCO Precis Oncol. 2018;2018.

148. Moreau P, Attal M, Hulin C, et al. Bortezomib, thalidomide, and dexamethasone with or without daratumumab before and after autologous stem-cell transplantaion for newly diagnosed multiple myeloma (CASSIOPEIA): a randomised, open-label, phase 3 study. Lancet. 2019;394(10192):2938.

149. Richardson PG, Bringhen S, Voorhees P, et al. Melflufen plus dexamethasone in relapsed and refractory multiple myeloma (O-12-M1): a multicentre, international, open-label, phase 1-2 study. Lancet Haematol. 2020;7(5):e395-e407.

150. Schjesvold F, Robak P, Pour L, Ascham J, Sonneveld P. OCEAN: a randomized phase III study of melflufen + dexamethasone to treat relapsed refractory multiple myeloma. Future Oncol. 2020;16(11):631-641.

haematological | 2020; 105(10)

2367 